\title{
Thank you to our reviewers 2017
}

The Editor would like to publicly acknowledge the people listed below who served as reviewers on the journal during 2017. Without their efforts, the quality of the journal could not be sustained.

Abe, Seiichiro
Afzal, Nadeem
Alberto Gonzalez-
Gonzalez, Jose
Allan, Phillip
Amornyotin, Somchai
Andreyev, Jervoise
Balderramo, Domingo
Basford, Pete
Beintaris, Iosif
Bollegala, Natasha
Bonder, Alan
Bourke, Michael J
Bramble, Mike
Brookes, Matthew
Brooks, Alenka
Bulat, Robert
Caddy, Grant
Chapman, Warren
Chattree, Amit
Ciclitira, Paul
Cives, Mauro
Coleman, Helen
Crooks, Colin John
Devarbhavi, Harshad
Dhar, Anjan
Dunn, Jason

Abe, Seiichiro

El Mekkaoui, A Epstein, Owen

Feuerstein, Joe

Graupera, Isabel

Grover, Samir

Guslandi, Mario

Haidry, Rehan

Halloran, $S$

Hawthorne, Antony

Barney

Haycock, Adam

Hayee, Bu' Hussain

Helbren, Emma

Hitchins, Charlotte

Hoque, Sami

Hudson, Mark

Iacucci, Marietta

Imison, Candace

Jensen, Henry

Johnson, Gavin

Katsanos, Konstantinos

Katukuri, Vinay

Kawano, Seiji

Keane, Margaret

Kedar, Archana

Kemp, Karen

Kim, Eun Soo
Koulaouzidis, Anastasios

Krishnareddy, Suneeta

Lahiff, Conor

Lai, Michelle

Lens, Sabela

Liu, Ken

Lobo, Alan

Logan, Robert

Londoño, Maria Carlota

Lung, Phillip

Maenhout, Annelies

Major, Giles

Mariño, Zoe

McLaughlin, John

Meade, Uchu

Medcalf, Lucy

Mendizabal, Manuel

Menon, Shyam

Menys, Alex

Mountford, Christopher

O'Toole, Aoiblinn

Olmedo, Martin

Paine, Peter

Phull, Perminder

Piraka, Cyrus

Pox, Christian

Pritchard, Mark
Probert, Chris

Ragunath, Krish

Rajendran, Arun

Sanders, David

Sarkar, Sanchoy

Seaward, Edward

Selvaggi, Francesco

Shaji, Sebastian

Siddiqui, Uzma

Snape, William

Sole, Cristina

Stein, Jurgen

Sweis, Rami

Tharian, Benjamin

Thoufeeq, Mo

Tozer, Phil

Trivedi, Hirsh

Tsochatzis, Emmanouil

Valentin-Lopez, Beatriz

Valori, Roland

Volonaki, Eleni

Walters, Julian

Williams, Jessica

Zarate, Natalia

Zhan, Tianzuo 